\title{
Die Transformation einer geschlossenen Kurve in eine Ellipse
}

\begin{abstract}
Wolfgang Schuster
Wolfgang Schuster wurde 1940 in Köln geboren. Er studierte katholische Theologie, Philosophie und Mathematik in Bonn und Innsbruck und promovierte 1970 im Fach Mathematik an der Universität Bonn. Anschliessend war er als wissenschaftlicher Assistent in Bonn und im Schuldienst tätig. Seit 1980 arbeitet er am Deutschen Institut für Fernstudienforschung an der Universität in Tübingen. In seiner Freizeit beschäftigt er sich mit Wittgenstein, Musil, Joyce und Proust, und auf der sportlichen Seite mit Drachenfliegen.
\end{abstract}

\section{Einleitung}

In [3] wird gezeigt, wie man durch sukzessive Anwendung einer endlichen Folge von Parallelogramm-Konstruktionen ein beliebiges Polygon in ein affin-reguläres überführen kann. Ein affin-reguläres Polygon ist das Bild eines regulären Polygons unter einer affinen Abbildung. Die Vorgehensweise dabei ist folgende: Ein Polygon betrachten wir als $n$-tupel $\mathbf{z}=\left(z_{0}, z_{1}, \ldots, z_{n-1}\right)$ komplexer Zahlen. Mit Hilfe einer ParallelogrammKonstruktion $P_{\rho}$ führen wir das Polygon $\mathbf{z}$ in ein Polygon $\mathbf{w}$ über. Die ParallelogrammKonstruktion $P_{\rho}$ wird durch einen reellen Streckungsfaktor $\rho \geq 0$ festgelegt: Man ergänzt die benachbarten Eckpunkte $z_{j-1}, z_{j}, z_{j+1}$ durch einen vierten Punkt $z_{j}^{\prime}=z_{j+1}+z_{j-1}-z_{j}$ zu einem Parallelogramm und trägt im Punkt $z_{j}$ den Vektor $\rho\left(z_{j}^{\prime}-z_{j}\right)$ an. Dessen Spitze markiert den Eckpunkt

$$
w_{j}=z_{j}+\rho\left(z_{j-1}-z_{j}+z_{j+1}-z_{j}\right)
$$

eines neuen Polygons $\mathbf{w}=\left(w_{0}, w_{1}, \ldots, w_{n-1}\right)=P_{\rho} \mathbf{z}$.

Führt man in jedem Eckpunkt eines Polygons eine im vorliegenden Beitrag näher beschriebene einfache Parallelogrammkonstruktion durch, so entsteht ein affin-reguläres Polygon, d.h. das affine Bild eines regulären Polygons. Ausgehend von dieser diskreten Überlegung entwickelt Wolfgang Schuster ein kontinuierliches Verfahren, von dem er anschliessend zeigt, dass es beliebige hinreichend reguläre, geschlossene Kurven in eine Ellipse überführt, also in das affine Bild eines Kreises. Ein schönes Beispiel für eine nicht auf der Hand liegende Übertragung vom Diskreten ins Kontinuierliche. ust 


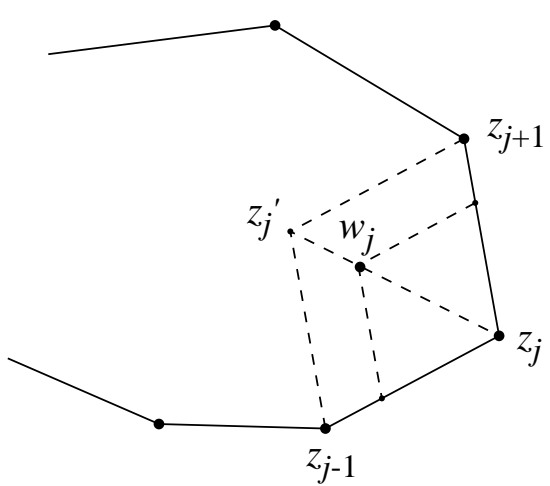

Abb. 1 Die Parallelogrammkonstruktion $P_{\rho}$.

Satz 1 Die Transformationen $P_{\rho_{k}}, \rho_{k}=1 /\left(4 \sin ^{2} \frac{\pi k}{n}\right), k=2,3, \ldots, \frac{1}{2}(n-1)$, führen ein beliebiges Polygon $\mathbf{z}$ mit ungerader Eckenzahl $n$ in ein affin-reguläres Polygon

$$
\mathbf{w}=P_{\rho_{2}} P_{\rho_{3}} \cdots P_{\rho_{\frac{1}{2}(n-1)}} \mathbf{z}
$$

über. Dabei ist die Reihenfolge der Transformationen $P_{\rho_{k}}$ beliebig.

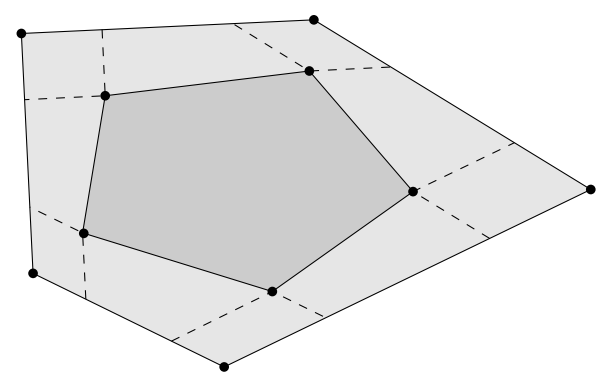

Abb. 2 Die Parallelogrammkonstruktion $P_{\rho}$ mit $\rho=\frac{1}{2}\left(1-\frac{1}{\sqrt{5}}\right)$ führt ein vorgegebenes Fünfeck in ein affin-reguläres Fünfeck über.

Die Faktoren $\rho_{k}$ besitzen eine geometrische Bedeutung: Die Länge der Diagonalen $\left(1, \lambda^{k}\right), \lambda=e^{\frac{2 \pi i}{n}}$, in dem von der $n$-ten Einheitswurzel $\lambda$ erzeugten regulären $n$-Eck $\left(1, \lambda, \lambda^{2}, \ldots, \lambda^{n-1}\right)$ ist $\left|1-\lambda^{k}\right|=2 \sin \frac{\pi k}{n}$.

Hat das Ausgangspolygon z gerade Eckenzahl, dann muß zu den Transformationen $P_{\rho_{k}}$, $k=2,3, \ldots, \frac{1}{2}(n-1)$, noch die Transformation $S$ hinzutreten, die ein Polygon $\mathbf{z}$ in das Polygon w der Seitenmitten $w_{j}=\frac{1}{2}\left(z_{j}+z_{j+1}\right)$ überführt. $S$ kann beim Anfangspolygon oder bei einem beliebigen Zwischenpolygon ausgeführt werden. Das Polygon $\mathbf{w}=P_{\rho_{2}} P_{\rho_{3}} \cdots P_{\rho_{\frac{1}{2}(n-1)}} S \mathbf{z}$ ist dann affin-regulär. Das Regularisierungsverfahren für Polygone übertragen wir auf eine Klasse hinreichend glatter geschlossener Kurven $z(t)$ der komplexen Ebene. 


\section{Erste heuristische Überlegung}

Durch eine periodische Funktion $z: \mathbb{R} \rightarrow \mathbb{C}$ mit Periode 1 wird in der Ebene $\mathbb{C}$ eine geschlossene Kurve mit der Punktmenge $\{z(t) \mid t \in[0,1]\}$ und der Parametrisierung $z$ gegeben; diese, die Punktmenge mit Parametrisierung, wird im folgenden mit $z(t)$ bezeichnet. Wir nehmen an, daß die Funktion $z$ an jeder Stelle $t \in \mathbb{R}$ in eine Taylorreihe entwickelt werden kann. Die obige Parallelogrammkonstruktion $P_{\rho_{k}}$ übertragen wir auf die Kurvenpunkte $z(t), z\left(t-\frac{1}{n}\right), z\left(t+\frac{1}{n}\right)$ mit $n \in \mathbb{N}$.

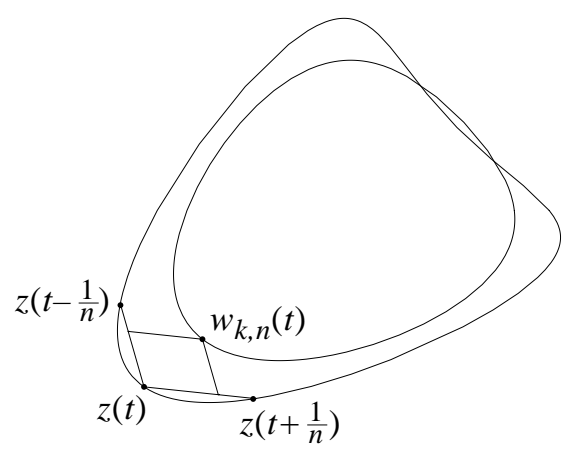

Abb. 3 Übertragung der Parallelogrammkonstruktion $P_{\rho}$ auf eine geschlossene Kurve $z(t)$.

Dadurch erhalten wir eine Kurve $w_{k, n}(t)$ mit

$$
w_{k, n}(t)=z(t)+\frac{1}{4 \sin ^{2} \frac{\pi k}{n}}\left(z\left(t-\frac{1}{n}\right)-z(t)+z\left(t+\frac{1}{n}\right)-z(t)\right) .
$$

Die Taylorentwicklung des Ausdrucks in der Klammer lautet:

$$
\begin{aligned}
z\left(t-\frac{1}{n}\right)+z\left(t+\frac{1}{n}\right)-2 z(t)= & z(t)-\frac{1}{n} z^{\prime}(t)+\frac{1}{2 n^{2}} z^{\prime \prime}(t) \\
& +z(t)+\frac{1}{n} z^{\prime}(t)+\frac{1}{2 n^{2}} z^{\prime \prime}(t)-2 z(t)+\cdots \\
= & \frac{1}{n^{2}} z^{\prime \prime}(t)+\cdots+\text { (Terme höherer Ordnung) } .
\end{aligned}
$$

Also gilt

$$
w_{k, n}(t)=z(t)+\frac{1}{4 n^{2} \sin ^{2} \frac{\pi k}{n}}\left(z^{\prime \prime}(t)+\cdots\right)
$$

Wegen

$$
\lim _{n \rightarrow \infty} n \sin \frac{\pi k}{n}=\pi k
$$

folgt

$$
w_{k}(t)=\lim _{n \rightarrow \infty} w_{k, n}(t)=z(t)+\frac{z^{\prime \prime}(t)}{(2 \pi k)^{2}}
$$


Dieses Ergebnis gibt Veranlassung, die Differentialoperatoren

$$
P_{k}=1+\frac{D^{2}}{(2 \pi k)^{2}}, \quad(D z)(t)=\frac{d z(t)}{d t}, \quad k=1,2, \ldots,
$$

einzuführen, die miteinander kommutieren. Es gilt dann

$$
w_{k}(t)=\left(\left(1+\frac{D^{2}}{(2 \pi k)^{2}}\right) z\right)(t)=\left(P_{k} z\right)(t)
$$

\section{Geometrische Deutung}

Die Transformation der Kurve $z(t)$ in die Kurve $w_{k}(t)$ läßt sich aufgrund der Zerlegung von $P_{k}$ in Linearfaktoren $P_{k}^{+}$und $P_{k}^{-}$geometrisch deuten. Offenbar ist

$$
P_{k}=1+\frac{D^{2}}{(2 \pi k)^{2}}=\left(1+\frac{i D}{2 \pi k}\right)\left(1-\frac{i D}{2 \pi k}\right)=P_{k}^{+} P_{k}^{-}
$$

Die Kurve

$$
z^{-}(t)=\left(P_{k}^{-} z\right)(t)=z(t)-\frac{i z^{\prime}(t)}{2 \pi k}
$$

ist die Ortskurve der Spitze des in den Kurvenpunkten $z(t)$ in bezug auf den Durchlaufsinn nach rechts angetragenen Normalenvektors $z^{\prime}(t) / 2 \pi k$. Entsprechend ist $z^{+}(t)$ die Ortskurve der Spitzen der nach links angetragenen Normalenvektoren.

Beispiel: Der Operator $P_{1}^{-}$transformiert die Ellipse

$$
z(t)=a \cos 2 \pi t+i b \sin 2 \pi t
$$

mit den beiden Halbachsen $a, b$ in die Kurve

$$
z^{-}(t)=(a+b)(\cos 2 \pi t+i \sin 2 \pi t)
$$

Das ist die Parameterdarstellung eines Kreises um den Mittelpunkt der Ellipse mit dem Radius $a+b$. Der Normalenvektor im Punkt $z(t)$ ist

$$
\frac{-i z^{\prime}(t)}{2 \pi}=i a \sin 2 \pi t+b \cos 2 \pi t=-i z\left(t+\frac{1}{4}\right) \text {. }
$$

Dieser geht also durch eine Drehung um $90^{\circ}$ im Uhrzeigersinn aus dem Vektor $z\left(t+\frac{1}{4}\right)$ hervor. Der Vektor $z\left(t+\frac{1}{4}\right)$ hat die Richtung der Tangente an die Ellipse im Punkt $z(t)$. D.h., $\left|\frac{i z^{\prime}(t)}{2 \pi}\right|$ ist gleich der Länge des halben Durchmessers, der zu dem durch $z(t)$ bestimmten Durchmesser der Ellipse konjugiert ist. - Damit haben wir den Satz von Napoleon-Barlotti für ein "affin-reguläres Polygon mit unendlicher Eckenzahl", das ist eine Ellipse, gewonnen (vgl. [2]). Die Anwendung des Differentialoperators $1+\frac{i D}{2 \pi}$ auf die Gleichung der Ellipse führt auf die Variante dieses Satzes, bei der die Normalenvektoren nach innen angetragen werden. 
Jede glatte 1-periodische Kurve $z(t)$ der komplexen Eben ist als Fourierreihe

$$
z(t)=a_{0}+\sum_{l \in \mathbb{N}}\left(a_{l} e^{2 \pi i l t}+a_{-l} e^{-2 \pi i l t}\right)
$$

mit Koeffizienten $a_{0}, a_{l}, a_{-l} \in \mathbb{C}$ darstellbar. Dabei kann der Summand

$$
e_{l}(t)=a_{l} e^{2 \pi i l t}+a_{-l} e^{-2 \pi i l t}, 0 \leq t \leq 1
$$

als Parameterdarstellung einer $l$-fach durchlaufenen Ellipse interpretiert werden.

Man erhält unmittelbar die Beziehungen

$$
\left(P_{k} e_{l}\right)(t)=\left(1-\frac{l^{2}}{k^{2}}\right) e_{l}(t)
$$

aus denen man für $k=l$ den Auslöschungseffekt $P_{k} e_{k}=0$ abliest.

Es ist daher zu vermuten, daß die Funktionenfolge

$$
z_{m}(t)=\left(P_{m} z_{m-1}\right)(t), m=2,3, \ldots
$$

mit $z_{1}(t)=z(t)$ für $m \rightarrow \infty$ gegen eine Ellipse $z_{\infty}(t)$ konvergiert, sofern nur die Anfangsfunktion $z(t)$ hinreichend glatt, d.h. hinreichend oft stetig differenzierbar, ist. In einem letzten Schritt kann $z_{\infty}(t)$ dann mit Hilfe des Operators $P_{1}^{-}$oder $P_{1}^{+}$(entsprechend dem Satz von Napoleon-Barlotti) in einen Kreis transformiert werden.

Die Vermutung ist sicher richtig, wenn die Reihe (5) endlich ist. Denn wenden wir auf

$$
z(t)=a_{0}+\sum_{l=1}^{n}\left(a_{l} e^{2 \pi i l t}+a_{-l} e^{-2 \pi i l t}\right)
$$

den Produktoperator

$$
T_{m}=\prod_{k=2}^{m} P_{k}=\prod_{k=2}^{m}\left(1+\frac{D^{2}}{(2 \pi k)^{2}}\right), m \geq 2,
$$

an, dann erhalten wir

$$
\left(T_{m} z\right)(t)=a_{0}+\prod_{k=2}^{m}\left(1-\frac{1}{k^{2}}\right)\left(a_{1} e^{2 \pi i t}+a_{-1} e^{-2 \pi i t}\right)
$$


denn es ist $\left(T_{m} e_{l}\right)(t)=0$ für $l \geq 2$. Bekanntlich ist

$$
\prod_{k=2}^{\infty}\left(1-\frac{1}{k^{2}}\right)=\frac{1}{2}
$$

also ergibt sich für $m \rightarrow \infty$ schließlich

$$
z_{\infty}(t)=a_{0}+\frac{1}{2}\left(a_{1} e^{2 \pi i t}+a_{-1} e^{-2 \pi i t}\right)
$$

das ist, bis auf den Faktor 1/2, die Anfangsellipse der Fourierentwicklung (8) von $z(t)$.

Ist die Reihe (5) nicht endlich, dann erhält man, sofern nur der Operator $T_{m}$ gliedweise angewendet werden darf,

$$
\begin{aligned}
\left(T_{m} z\right)(t)=a_{0} & +\prod_{k=2}^{m}\left(1-\frac{1}{k^{2}}\right)\left(a_{1} e^{2 \pi i t}+a_{-1} e^{-2 \pi i t}\right) \\
& +\sum_{l=m+1}^{\infty} \prod_{k=2}^{m}\left(1-\frac{l^{2}}{k^{2}}\right)\left(a_{l} e^{2 \pi i l t}+a_{-l} e^{-2 \pi i l t}\right) .
\end{aligned}
$$

Wegen $\prod_{k=2}^{\infty}\left(1-\frac{1}{k^{2}}\right)=\frac{1}{2}$ muß sich die Überlegung zur Konvergenz der Funktionenfolge $z_{m}(t)=\left(T_{m} z\right)(t)$ nur noch um das Verhalten des Restgliedes

$$
R_{m}(t)=\sum_{l=m+1}^{\infty} \prod_{k=2}^{m}\left(1-\frac{l^{2}}{k^{2}}\right)\left(a_{l} e^{2 \pi i l t}+a_{-l} e^{-2 \pi i l t}\right)
$$

kümmern (s. Abschnitt 3).

\section{Zweite heuristische Überlegung}

Wir berechnen im folgenden den Grenzwert $T_{\infty}=\lim _{m \rightarrow \infty} T_{m}$ der Operatorenfolge $T_{m}$ und bestimmen die Funktion $z_{\infty}=T_{\infty} z$. Ohne daß wir auf die Fourieranalyse von $z$ Bezug nehmen, ergibt sich dann direkt: $z_{\infty}$ ist die Parameterdarstellung einer Ellipse.

Aus der Produktdarstellung der Sinusfunktion

$$
\frac{1}{2 i}\left(e^{i \pi x}-e^{-i \pi x}\right)=\sin \pi x=\pi x \prod_{k=1}^{\infty}\left(1-\frac{x^{2}}{k^{2}}\right)
$$

ergibt sich mit $i \pi x=\frac{y}{2}$ die Beziehung

$$
e^{\frac{y}{2}}-e^{-\frac{y}{2}}=y \prod_{k=1}^{\infty}\left(1-\frac{y^{2}}{(2 \pi k)^{2}}\right),
$$


die mit $y=D$ in die Operatorgleichung

$$
e^{\frac{D}{2}}-e^{-\frac{D}{2}}=D \prod_{k=1}^{\infty}\left(1-\frac{D^{2}}{(2 \pi k)^{2}}\right)
$$

übergeht. Darin ist das unendliche Produkt rechts durch den Grenzwert

$$
P z=\lim _{m \rightarrow \infty} \prod_{k=1}^{m}\left(1+\frac{D^{2}}{(2 \pi k)^{2}}\right) z
$$

erklärt, dessen Existenz wir für eine gewisse Klasse von Funktionen $z$ hier voraussetzen und im folgenden Abschnitt beweisen wollen. (Auf einen strikten Beweis der Operatorgleichung (13) wollen wir hingegen verzichten. Er ließe sich z.B. mit Hilfe der Laplace-Transformation führen. Man hat dann zu zeigen, daß die Funktionen $T_{1} z$ und $T_{2} z$ dieselbe Laplace-Transformierte besitzen, wenn $T_{1}$ der bei (13) links stehende und $T_{2}$ der rechts stehende Operator ist und die Funktion $z$ im Durchschnitt der Definitionsbereiche von $T_{1}$ und $T_{2}$ liegt.) Der Operator $e^{c D}, c \in \mathbb{R}$, ist durch die Exponentialreihe

$$
\left(e^{c D} z\right)(t)=\sum_{k=0}^{\infty}\left(\frac{(c D)^{k}}{k !} z\right)(t)=\sum_{k=0}^{\infty} \frac{c^{k}}{k !} z^{(k)}(t)
$$

erklärt. Hat $z(t)$ eine überall konvergente Potenzreihenentwicklung, so gewinnt man nach einfacher Rechnung:

$$
\left(e^{c D} z\right)(t)=z(t+c)
$$

Erfüllt die Funktion $w$ die Beziehung

$$
\left(D\left(1+\frac{D^{2}}{(2 \pi)^{2}}\right)\right) w=z
$$

d.h., ist $w$ eine Lösung der Differentialgleichung

$$
w^{\prime}(t)+\frac{w^{\prime \prime \prime}(t)}{(2 \pi)^{2}}=z(t)
$$

dann erhält man wegen der Vertauschbarkeit der Operatoren $D$ und $P_{k}$ für den in (9) definierten Operator $T_{m}$

$$
T_{m} z=\left(D\left(1+\frac{D^{2}}{(2 \pi)^{2}}\right) T_{m}\right) w=\left(D \prod_{k=1}^{m}\left(1+\frac{D^{2}}{(2 \pi k)^{2}}\right)\right) w,
$$

und durch Grenzübergang $m \rightarrow \infty$ folgt mit (13)

$$
T_{\infty} z=\left(e^{\frac{D}{2}}-e^{-\frac{D}{2}}\right) w
$$


Bezeichnet man mit $L$ den zu (15) gehörigen Lösungsoperator, das ist die Abbildung $L$, die jeder Funktion $z$ die Menge $L(z)$ der zugehörigen Lösungen $w$ von (15) zuordnet, dann läßt sich der Grenzoperator $T_{\infty}$ in der Form

$$
T_{\infty}=\left(e^{\frac{D}{2}}-e^{-\frac{D}{2}}\right) L
$$

notieren. Diese Schreibweise kann man folgendermaßen rechtfertigen: Als Bild der Menge $L(z)$ unter der Abbildung $e^{D / 2}-e^{-D / 2}$ definieren wir die Funktion $\left(e^{D / 2}-\right.$ $\left.e^{-D / 2}\right) w$, wobei $w$ ein beliebiges Element aus $L(z)$ ist. Mit (14) ergibt sich also (in Übereinstimmung mit (16)):

$$
\left(T_{\infty} z\right)(t)=w\left(t+\frac{1}{2}\right)-w\left(t-\frac{1}{2}\right) .
$$

Diese Definition des Operators $T_{\infty}$ ist eindeutig, also unabhängig von der speziellen Wahl der Lösung $w \in L(z)$. Ist nämlich $w_{1} \in L(z)$ eine andere Lösung, dann gilt $w_{1}=w+h$, wobei $h(t)=c_{1} e^{2 \pi i t}+c_{2} e^{-2 \pi i t}+c_{3}, c_{1}, c_{2}, c_{3} \in \mathbb{C}$, eine Lösung der zu (15) gehörigen homogenen Differentialgleichung ist. Die Funktion $h$ ist 1-periodisch. Es folgt daher $h\left(t+\frac{1}{2}\right)-h\left(t-\frac{1}{2}\right)=0$, also $w\left(t+\frac{1}{2}\right)-w\left(t-\frac{1}{2}\right)=w_{1}\left(t+\frac{1}{2}\right)-w_{1}\left(t-\frac{1}{2}\right)$.

Ist nun $Z$ eine Stammfunktion von $z$, dann ist

$$
w(t)=Z(t)-\int_{0}^{t} \cos 2 \pi(t-\tau) z(\tau) d \tau
$$

eine Lösung von (15), wie man leicht nachrechnet. Mit (18) ergibt sich also

$$
\begin{aligned}
\left(T_{\infty} z\right)(t)=\int_{t-\frac{1}{2}}^{t+\frac{1}{2}} z(\tau) d \tau & -\int_{0}^{t+\frac{1}{2}} \cos 2 \pi\left(t+\frac{1}{2}-\tau\right) z(\tau) d \tau \\
& +\int_{0}^{t-\frac{1}{2}} \cos 2 \pi\left(t-\frac{1}{2}-\tau\right) z(\tau) d \tau
\end{aligned}
$$

Die Integranden sind 1-periodische Funktionen, so daß mit $T_{\infty} z=z_{\infty}$ weiter gilt:

$$
\begin{aligned}
z_{\infty}(t) & =\int_{0}^{1} z(\tau) d \tau+\int_{0}^{1} \cos 2 \pi(t-\tau) z(\tau) d \tau \\
& =\int_{0}^{1} z(\tau) d \tau+\frac{1}{2} \int_{0}^{1} e^{-2 \pi i \tau} z(\tau) d \tau \cdot e^{2 \pi i t}+\frac{1}{2} \int_{0}^{1} e^{2 \pi i \tau} z(\tau) d \tau \cdot e^{-2 \pi i t}
\end{aligned}
$$

Mit den Koeffizienten

$$
a_{0}=\int_{0}^{1} z(\tau) d \tau, a_{1}=\int_{0}^{1} e^{-2 \pi i \tau} z(\tau) d \tau, a_{-1}=\int_{0}^{1} e^{2 \pi i \tau} z(\tau) d \tau
$$

erhalten wir also wieder die Grenzkurve

$$
z_{\infty}(t)=a_{0}+\frac{1}{2}\left(a_{1} e^{2 \pi i t}+a_{-1} e^{-2 \pi i t}\right) .
$$

Die Zahlen $a_{0}, a_{1}, a_{-1}$ sind die ersten Koeffizienten der Fourierentwicklung der Funktion $z$. 


\section{Eine Konvergenzbedingung}

Die Funktion $f$ sei in einem Gebiet holomorph, das einen Kreisring $K_{R}=\left\{z \mid R^{-1} \leq\right.$ $|z| \leq R\}$ mit $R>1$ enthält. Sie kann dann dort in eine Laurentreihe

$$
f(z)=a_{0}+\sum_{l \in \mathbb{N}}\left(a_{l} z^{l}+a_{-l} z^{-l}\right)
$$

entwickelt werden. Für die Koeffizienten $a_{l}, a_{-l}$ dieser Entwicklung gilt eine Abschätzung der Form (vgl. [1], S. 189 ff.)

$$
\left|a_{l}\right|,\left|a_{-l}\right| \leq \frac{M}{R^{l}},
$$

wenn $M>0$ eine obere Schranke für $|f(z)|, z \in K_{R}$, darstellt. Wir betrachten das Bild des Einheitskreises unter der Abbildung $f$, das durch die 1-periodische Funktion $z$ mit

$$
z(t)=a_{0}+\sum_{l \in \mathbb{N}}\left(a_{l} e^{2 \pi i l t}+a_{-l} e^{-2 \pi i l t}\right), t \in \mathbb{R}
$$

parametrisiert wird. Da die Reihe (22) beliebig oft gliedweise differenziert werden darf, darf der Operator $T_{m}$ auf die Entwicklung (24) gliedweise angewendet werden. Die Funktionenfolge $T_{m} z$ konvergiert also genau dann gegen eine Grenzfunktion $z_{\infty}$, wenn das Restglied (12) für $m \rightarrow \infty$ gegen Null geht. Mit (23) erhalten wir zunächst die Abschätzung

$$
\left|R_{m}(t)\right| \leq \sum_{l=m+1}^{\infty}\left|\prod_{k=2}^{m}\left(1-\frac{l^{2}}{k^{2}}\right)\right| \frac{2 M}{R^{l}}
$$

für den in (12) definierten Term. Wir betrachten daher den Ausdruck

$$
\left|\prod_{k=2}^{m}\left(1-\frac{l^{2}}{k^{2}}\right)\right|=\frac{1}{(m !)^{2}} \prod_{k=2}^{m}(l-k)(l+k) .
$$

Zwischen arithmetischem und geometrischem Mittel positiver Zahlen $a_{1}, a_{2}, \ldots, a_{n}$ besteht die Ungleichung

$$
\left(a_{1} \cdot a_{2} \cdots \cdot a_{n}\right)^{\frac{1}{n}} \leq \frac{1}{n}\left(a_{1}+a_{2}+\cdots+a_{n}\right),
$$

also

$$
a_{1} \cdot a_{2} \cdots a_{n} \leq n^{-n}\left(a_{1}+a_{2}+\cdots+a_{n}\right)^{n} .
$$

Daher gilt

$$
\prod_{k=2}^{m}(l-k)(l+k) \leq l^{2 m-2}
$$

also

$$
\left|R_{m}(t)\right| \leq \frac{2 M}{(m !)^{2}} \sum_{l=m+1}^{\infty} \frac{l^{2 m-2}}{R^{l}} .
$$


Die Funktion $g$ mit $g(x)=x^{2 m-2} R^{-x}, m \geq 2, x \geq 0$, hat ihr Maximum bei $x_{0}=$ $(2 m-2) / \log R$ und fällt streng mononton für $x \leq x_{0}$. Ist $\log R \geq 2$, also $R \geq e^{2}$, dann ist $x_{0}<m$, so daß gilt

$$
\sum_{l=m+1}^{\infty} \frac{l^{2 m-2}}{R^{l}}<\int_{0}^{\infty} x^{2 m-2} R^{-x} d x
$$

da die links stehende Summe als Summe von Rechteckflächen gedeutet werden kann, die unterhalb des Graphen von $g$ liegen. Das Integral kann man dann mit Hilfe der Transformation $t=x \log R$ auf die Integraldarstellung der Gammafunktion zurückführen:

$$
\int_{0}^{\infty} x^{2 m-2} R^{-x} d x=\frac{1}{(\log R)^{2 m-1}} \int_{0}^{\infty} t^{2 m-2} e^{-t} d t=\frac{(2 m-2) !}{(\log R)^{2 m-1}} .
$$

Damit erhalten wir die Abschätzung

$$
\left|R_{m}(t)\right| \leq \frac{(2 m-2) ! 2 M}{(m !)^{2}(\log R)^{2 m-1}}
$$

und wegen $R \geq e^{2}$, d.h. $\log R \geq 2$, folgt

$$
\left|R_{m}(t)\right| \leq \frac{(2 m-2) ! 4 M}{(m !)^{2} 2^{2 m}} .
$$

Mit Hilfe der Stirlingschen Formel $n !=n^{n} e^{-n} \sqrt{2 \pi n} e^{\frac{\vartheta}{12 n}}, 0<\vartheta<1$, ergibt sich daraus schließlich die Abschätzung

$$
\left|R_{m}(t)\right| \leq \frac{M}{(m-1)^{2} \sqrt{m}} .
$$

Diese Abschätzung kann sicherlich noch verbessert werden. Sie soll hier nur dem Zweck dienen, eine hinreichende Bedingung für $\lim _{m \rightarrow \infty} R_{m}(t)=0 \mathrm{zu}$ formulieren.

Damit ist gezeigt, daß die Klasse der Funktionen $z$, die zu einer konvergenten Funktionenfolge $T_{m} z$ Anlaß geben, umfassender ist als die Klasse der Funktionen $z$ mit einer endlichen Fourierentwicklung. - Unser Ergebnis fassen wir zusammen:

Satz 2 Die Funktion $f$ sei holomorph in einem Gebiet, das den Kreisring $K_{e^{2}}=\left\{z \mid e^{-2} \leq\right.$ $\left.|z| \leq e^{2}\right\}$ enthält, und

$$
f(z)=a_{0}+\sum_{l \in \mathbb{N}}\left(a_{l} z^{l}+a_{-l} z^{-l}\right)
$$

sei ihre Laurentreihe. Ist dann $z(t)=f\left(e^{2 \pi i t}\right), t \in[0,1]$, das Bild des Einheitskreises unter der Abbildung $f$, dann konvergiert die Funktionenfolge $T_{m} z, m \geq 2$, mit

$$
T_{m}=\prod_{k=2}^{m}\left(1+\frac{D^{2}}{(2 \pi k)^{2}}\right),(D z)(t)=\frac{d z(t)}{d t},
$$

gleichmäßig in $[0,1]$ gegen die Funktion $z_{\infty}$ mit

$$
z_{\infty}(t)=a_{0}+\frac{1}{2}\left(a_{1} e^{2 \pi i t}+a_{-1} e^{-2 \pi i t}\right) .
$$

Der Graph der Funktion $z_{\infty}$ ist eine Ellipse. 

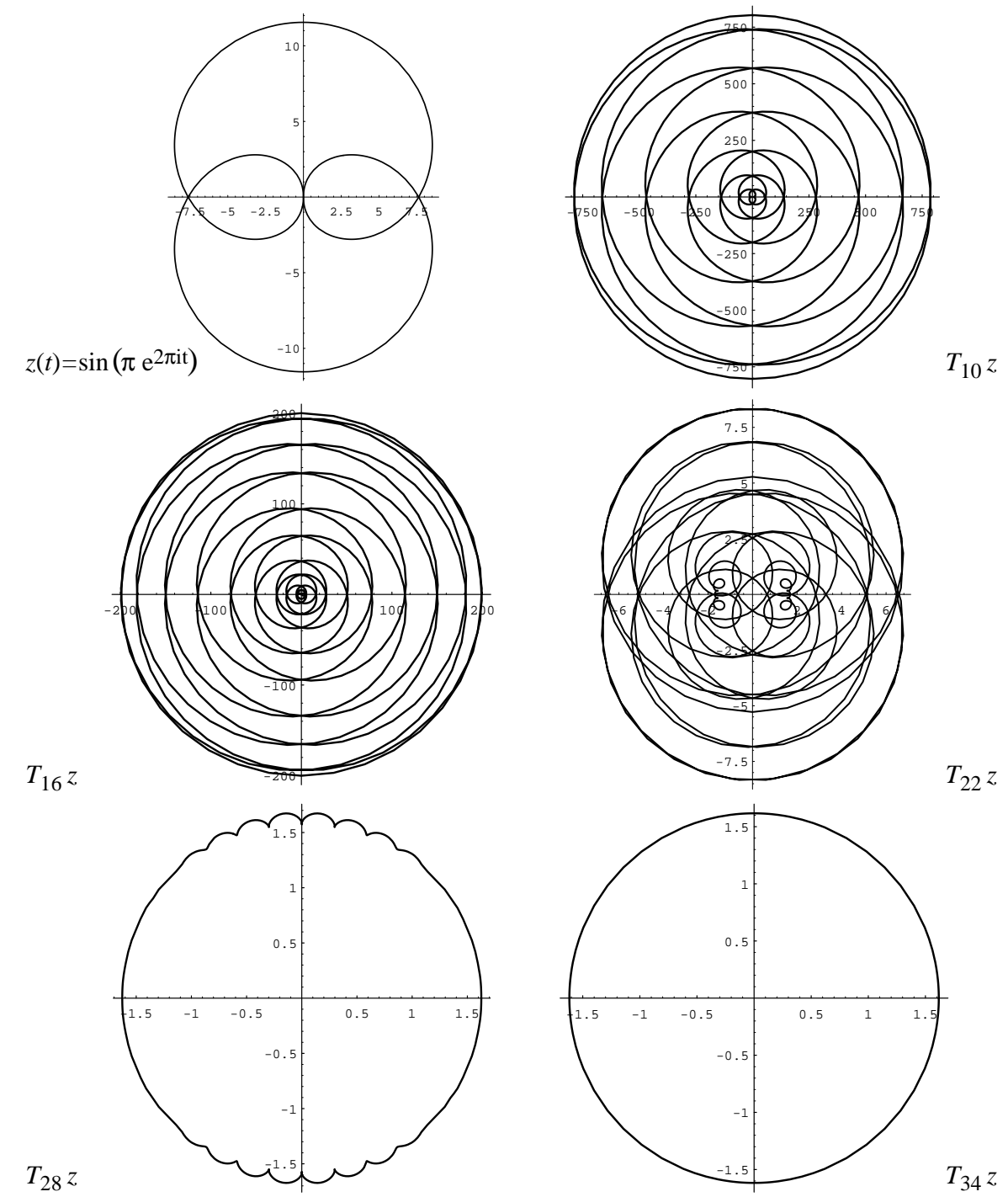

Abb. 4 Transformation der Kurve $z(t)=\sin \left(\pi e^{2 \pi i t}\right)$ in den Kreis $z_{\infty}(t)=\frac{1}{2} \pi e^{2 \pi i t}$. Man beachte das explosionsartige Größenwachstum bei den Anfangsgliedern der Folge $T_{m}^{2} z$.

Bemerkung: Es mag von einem geometrischen Standpunkt aus unbefriedigend erscheinen, daß die Transformation einer geschlossenen Kurve $z(t)$ in eine Ellipse auf eine spezielle Parametrisierung der Kurve Bezug nimmt. Die Länge der anzutragenden Normalenvektoren ändert sich dann im allgemeinen mit dem Kurvenpunkt. Daß die Transformation einer geschlossenen Kurve in eine Ellipse auch durch Antragen von Normalenvektoren gleicher Länge möglich ist, zeigt man mit Hilfe der natürlichen Parametrisierung durch die Bogenlänge $s$. Die Funktion $z$ hat dann die Periode $L$, wenn $L$ die Länge der Kurve 
ist. Besitzt $z$ eine Fourierentwicklung

$$
z(s)=b_{0}+\sum_{l \in \mathbb{N}}\left(b_{l} e^{\frac{2 \pi i l s}{L}}+b_{-l} e^{\frac{-2 \pi i l s}{L}}\right)
$$

so transformiert die Folge der Differentialoperatoren

$$
P_{k}=1+\frac{(L D)^{2}}{(2 \pi k)^{2}}=\left(1+\frac{i L D}{2 \pi k}\right)\left(1-\frac{i L D}{2 \pi k}\right)
$$

die Kurve $z(s)$ in die Ellipse

$$
z_{\infty}(s)=b_{0}+\frac{1}{2}\left(b_{1} e^{\frac{2 \pi i s}{L}}+b_{-1} e^{-\frac{2 \pi i s}{L}}\right)
$$

sofern $z(s)$ eine hinreichend glatte Kurve ist. Wegen $\left|z^{\prime}(s)\right|=1$ ist die Länge $L / 2 \pi k$ der Normalenvektoren jetzt unabhängig von dem Kurvenpunkt, an dem sie angetragen werden.

Für die kritische Durchsicht des Manuskripts und wertvolle Verbesserungsvorschläge danke ich Herrn Günter Pickert (Gießen). Herrn Carsten Kühn danke ich für die sorgfältige Herstellung des Textes und der Abbildungen.

\section{Literatur}

[1] H. Behnke, F. Sommer: Theorie der analytischen Funktionen einer komplexen Veränderlichen. 3. Auflage. Springer-Verlag, Berlin, Heidelberg, New York 1972.

[2] R. Fritsch: Bemerkungen zum Satz von Napoleon-Barlotti im Unterricht. In: Mathematik erfahren und lehren. Festschrift für H.-J. Vollrath, herausgegeben von G. Pickert und I. Weidig. Ernst Klett Schulbuchverlag, Stuttgart 1994.

[3] W. Schuster: Regularisierung von Polygonen. Math. Semesterber. 45 (1998), 77-94.

Wolfgang Schuster

Deutsches Institut für Fernstudienforschung (DIFF)

an der Universität Tübingen

Konrad-Adenauer-Str. 40

D-72072 Tübingen 